\title{
The Potential for Energy Reduction in UK Commercial Offices Through Effective Management and Behaviour Change
}

\author{
Mark Mulville \\ Technological University Dublin (formerly Uni. of Greenwich), mark.mulville@tudublin.ie \\ Keith Jones \\ Anglia Ruskin University \\ Gesche Huebner \\ University College London
}

Follow this and additional works at: https://arrow.tudublin.ie/beschrecart

Part of the Architectural Engineering Commons, and the Social and Behavioral Sciences Commons

\section{Recommended Citation}

Mulville, M., Jones, K. \& Huebner, G. (2014). The potential for energy reduction in UK commercial offices through effective management and behaviour change. Journal of Architectural Engineering and Design Management, Vol. 10, 1-2, pp.79-90, 2014. doi:10.1080/17452007.2013.837250

This Article is brought to you for free and open access by the School of Surveying and Construction Management at ARROW@TU Dublin. It has been accepted for inclusion in Articles by an authorized administrator of ARROW@TU Dublin. For more information, please contact arrow.admin@tudublin.ie, aisling.coyne@tudublin.ie, gerard.connolly@tudublin.ie.

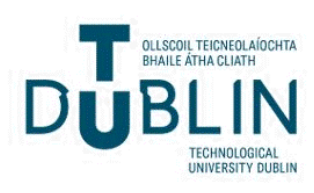




\section{The potential for energy reduction in UK commercial offices through effective management and behaviour change.}

Mark Mulville (contact for correspondence)

Department of Property and Construction Management, School of Architecture, Design and Construction, University of Greenwich

Mansion Site, Bexley Road,

London, SE9 2PQ,

$U K$.

Phone: 02083319973

m.mulville@gre.ac.uk

Keith Jones

Department of Property and Construction Management, School of Architecture, Design and Construction,

University of Greenwich

Mansion Site, Bexley Road,

London, SE9 $2 P Q$,

$U K$.

Phone: 02083319260

k.g.jones@gre.ac.uk

Gesche Huebner ${ }^{1}$

Department of Property and Construction Management, School of Architecture, Design and Construction,

University of Greenwich

Mansion Site, Bexley Road,

London, SE9 2PQ,

$U K$.

Phone: 02083319100

g.m.huebner@gre.ac.uk

\footnotetext{
${ }^{1}$ Now of University College London (UCL) Energy Institute.
} 


\section{The potential for energy reduction in UK commercial offices through effective management and behaviour change.}

General office equipment can be responsible for a significant proportion of overall electrical energy consumption in UK offices and this is predicted to rise significantly over the coming years (Webber et al. 2001). As a significant contributor to overall energy use, this equipment has a corresponding cost and carbon impact. The legally binding targets of The Climate Change Act (2008) present a need to address the impact of office equipment, especially within the less efficient existing building stock. In this study the range of energy consumption patterns observed across workstations in two typical UK, air-conditioned office spaces covering 90 desks, and the potential effect of using feedback to encourage energy reduction through behaviour change is explored. The project monitored energy consumption over a four month baseline period. Taking into account technical, behavioural and environmental influences this baseline established that a significant variation in consumption patterns exists between workstations providing the same function, in comparable locations and over the same period of time. Following the establishment of the baseline data further monitoring took place over the following period to assess the effect of behaviour change interventions through the provision of comparative feedback. The core driver behind the variation in consumption identified was found to be occupant behaviour over technical and environmental considerations. The study establishes that it is possible to reduce energy use, carbon emissions and cost associated with desk level electricity consumption by up to $20 \%$ through behaviour change in typical UK office spaces. Further savings are possible through energy management and procurement policy, but behaviour change offers significant initial reductions for limited investment.

Keywords: energy, small power, office, $\mathrm{CO} 2$, unregulated energy, feedback, behaviour change. 


\section{Introduction:}

The impact and pattern of usage relating to regulated energy within the building stock is generally well understood. Consumption patterns associated with unregulated energy such as small power and desktop equipment is less well understood. Junnila (2007) notes that the existing literature on energy efficiency in office buildings does not provide good data for estimating energy reduction potential through occupant behaviour change. Furthermore as discussed by Menezes, Cripps, Bouchlaghem and Buswell (2012) this lack of understanding of unregulated energy use can be identified as a contributory factor to the 'performance gap' between predicted/design and actual/in-use energy performance. In UK office spaces this performance gap has in some cases resulted in in-use carbon emissions 2-3 times that of the original design estimate (Bordass, Cohen \& Field, 2004). The UK target of a 34\% reduction in $\mathrm{CO}_{2}$ emissions over 1990 levels by 2020 (The Climate Change Act, 2008) has resulted in the construction industry introducing increasingly stringent energy performance regulations. As building regulations and other statutory mechanisms drive down the consumption associated with regulated energy, an understanding of unregulated energy becomes increasingly important. Information and communication technology (ICT) can be responsible for $20 \%$ or more (Chartered Institution of Building Services Engineers [CIBSE], 2004) of overall electrical energy consumption in a typical office space. Improvements in the energy efficiency of I.T equipment have reduced consumption of individual units. Conversely increased processing power and range of equipment utilised has resulted in predictions that small power will continue to have a significant impact (Jenkins, Singh \& Eames, 2009) and that energy consumption associated with office eqiupment will continue to grow globally in the near future. (Webber et al. 2001 \& Vereecken et al. 2010.) 
As noted by Junnila (2007) few studies have focused on quantifying the enduser influence on energy consumption, furthermore most energy managers believe end users influence to be minimal (Lukas, 2000). However it has previously been established that energy use of desktop equipment is highly influenced by occupant behaviour and is flexible in nature (Zhang, Siebers \& Aickelin, 2011). This view is supported by a study by Kawamoto, Shimoda and Mizuno (2003) which estimated that for an average working day the actual in-use utilisation of desktop equipment may commonly be as low as $43 \%$. Additionally many office workers don't power down equipment at the end of the working day (Berl \& de Meer 2011) and even fewer unplug equipment that may still draw power when turned off. A US field survey of office equipment operating patterns (Webber et al. 2001) found that only $44 \%$ of computers and $32 \%$ of monitors where turned off at night, a similar UK based study (Zhang et al. 2011) found that $60 \%$ of occupants don't power down at night time, with $31 \%$ powering down just occasionally and only $9 \%$ powering down regularly. Comparison of these two studies would seem to suggest that organisational or cultural background may have an influence on the rate of power down and reinforces the view that small power consumption patterns are generally not fully understood but offer significant potential for savings.

The flexible nature of desktop energy consumption supports the view that there is significant potential for energy reduction through behaviour change. Ward (2008) identifies increasing IT usage in commercial offices as one of the main barriers to reducing energy use, however if occupant behaviour were better understood it may be possible to overcome such barriers while allowing for the increased use of I.T. equipment. There is evidence that the use of behaviour change mechanisms can 
significantly reduce overall energy consumption in commercial buildings. It has been estimated that workstation energy use can account for $73-88 \%$ of total office equipment energy use and that a combination of behaviour change, energy management and procurement policy could contribute to a $60-80 \%$ reduction in this (Junnila, 2007). With this in mind a focus on desk level equipment would appear valid when considering energy reduction from general office equipment. Carrico and Riemer (2011) in a study considering university based office spaces in the US, note that energy use reductions of up to $15 \%$ should be possible through the implementation of behaviour change measures alone, by using group level feedback and peer education.

Jenkins et al. (2009) predict that offices spaces in London and the south of the UK are likely to increasingly tend towards being cooling dominated. As, in most UK offices, internal heat gains are the main contributing factor to cooling loads, the reduction of consumption associated with desktop equipment and corresponding heat gains could offer an indirect benefit by decreasing the corresponding cooling load (Jenkins, Liu, \& Peacock, 2007).

This study considers energy consumption patterns across 90 desks in two air conditioned offices spaces in South-East England. The variation in consumption patterns identified is considered and analysed, and a series of behaviour change interventions are made with the goal of reducing consumption associated with desktop equipment by at least $20 \%$. 


\section{Methodology}

Over the course of an unoccupied weekend 90 work stations (each workstation consists of a single screen, desktop computer or laptop and docking station, with two desk level plugs) were fitted with energy monitoring devices. The devices look like standard extension leads with four plug locations. Computers, screens, a desk level plug and any other desktop devices present were plugged into the monitoring device. The device was installed so that it became the most accessible plug point for the occupant at each work station. There were other plug locations available (from which the monitoring device itself is plugged) but these were out of sight and hidden within cable trays to the underside of the desk. This approach ensured all small power consumed at desk level could be monitored. The device used takes an hourly reading, which is then wirelessly transmitted to be stored on a central server in the office of the monitor provider, data can then be downloaded remotely for analysis. The device itself was tested and found to draw 0.8 watts which was accounted for in the discussions to follow.

The initial baseline monitoring period began and ended on the same day in both locations, lasting from the $17^{\text {th }}$ of June 2011 to $26^{\text {th }}$ of September 2011 (100 days). Following the conclusion of the baseline period a series of field surveys were conducted to identify which pieces of equipment were plugged into which monitor and to ensure that only monitors with a full profile (monitoring at least the screen and computer at the location) were taken forward for analysis. Additionally, field surveys were able to identify where additional equipment such as mobile phone chargers, other personal ICT devices, desk fans, desk lamps and heaters had been plugged in. Of the additional small power devices identified the most prevalent was mobile phone chargers with $21 \%$ of all 
work stations having chargers plugged in during the survey (many without a mobile phone connected).

Using the baseline data it was possible to establish total, daily and hourly consumption patterns at both site-wide and individual work station levels. Allowance was made for absence from the workplace through filtering the data to only cover days when the location was occupied. Further corrections where made to ensure readings from individual work stations where not skewed due to longer working hours. This was achieved through establishing an out of hours baseline power density (the average power density at the desk location when not in-use) and an operational power density (the average power density at the desk during operating hours). From this it was possible to ensure feedback provided made allowance for extended working hours without unduly penalising the work station occupant. This was achieved by applying average working and non-working hours based on site-wide data as opposed to individual usage, thus allowing like for like comparison. The baseline analysis identified a small number of unexpected peaks in energy use, based on the timing and frequency of the peaks they were attributed to the cleaning cycle at each location (see Figures $1 \& 2)$.

Once the baseline data was analysed it was compared to widely accepted industry benchmarks to understand the impact of small power within the benchmarks. For office location A it was possible to compare this back to overall electricity consumption for the site and produce $/ \mathrm{m}^{2}$ comparisons. It was not possible to indentify the same data for office location B as the data could not be disaggregated; therefore any such comparisons are based only on the data from office location A. 
As the target of the study was to understand variation in energy consumption and the influence of occupant behaviour, it was important to take account of ongoing changes within the office structure (turnover of staff, change of location) so that a clear picture of behaviour could be established. Working with the management team at both locations, in addition to the follow up field surveys, it was possible to ensure that only desks where the individual could be followed were included in the monitoring. This reduced the number of desks considered for post intervention analysis.

Following the baseline period a number of interventions were made prior to and during the follow up monitoring period. Interventions began in early March 2012 and continued until the end of June. Two types of feedback interventions were used in conjunction with goal-setting. Comparative feedback interventions considering the consumption of an individual or a group in relation to an average have been successful in reducing energy consumption in households, as has historic feedback (Abrahamse, Steg, Vlek \& Rothengatter, 2005). The basic idea of comparative feedback is to evoke a feeling of competition, social comparison, or social pressure which then leads to a changed behaviour to correspond more closely to the norm. In order to prevent a pull towards the mean performance in those who already show the desired behaviour (in this case, below-average energy use), an appraisal of the behaviour is employed, often in the form of a negative or positive smiley face. The first intervention consisted of providing regular historic (approximately every fortnight) feedback on energy consumption on an office-based level. This was combined with goal-setting; hence, at the beginning of the intervention, a reduction of $20 \%$ was calculated and given as a target. The second intervention was conducted with individual level feedback to half of the occupants within an office and group-based feedback to the other half. Consumption was split up 
in to a day and night-time component in order to highlight the wastage associated with leaving equipment on standby overnight.

Comparing the data generated from the follow up monitoring period it was possible to quantify the impact and longevity of the interventions made.

\section{Benchmark Comparison}

There is a wide range of industry benchmarks relating to regulated energy, unregulated energy, small power loads, occupant density and other related metrics. These are supported and supplemented by academic research and field studies. An understanding of these benchmarks can help to gauge the overall impact of workstation energy use patterns, in relation to the overall energy use framework. The figures discussed below feed into the floor and desk level analysis which follows.

Technical Memorandum 46 (TM46) (CIBSE, 2008) provides widely recognised energy benchmarks for UK buildings, however the $95 \mathrm{kWh} / \mathrm{m}^{2} / \mathrm{yr}$. for typical electrical energy consumption in UK offices identified within TM46 includes only regulated energy use. Therefore it is necessary to look to Energy Consumption Guide 19 (ECG19) (Department of the environment, transport and the regions [DETR], 2003.) which identifies a regulated electrical energy usage of $145 \mathrm{kWh} / \mathrm{m}^{2} / \mathrm{yr}$. in air conditioned offices with an additional $63 \mathrm{kWh} / \mathrm{m}^{2} / \mathrm{yr}$ allowed for unregulated energy of which $31 \mathrm{kWh} / \mathrm{m}^{2} / \mathrm{yr}$. is attributed to office equipment. If, as identified by Junnila (2007), 73$88 \%$ of this relates to desktop equipment, office equipment would typically be expected to consume $22-27 \mathrm{kWh} / \mathrm{m}^{2} / \mathrm{yr}$. the Chartered Institution of Building Services Engineers (CIBSE) Guide F (CIBSE, 2004) further supplements this discussion by identifying office equipment as being responsible for $20 \%$ of overall electrical energy consumption 
with two thirds of this attributed to desktop equipment. Applying this to the ECG19 benchmark, desktop energy usage can be calculated as $27.45 \mathrm{kWh} / \mathrm{m}^{2} / \mathrm{yr}$. aligning well with the findings of Junnila (2007). Good practice guide 11 (The Department of the Environment [DOE], 1996) would seem to contradict these predictions with a much more significant proportion of electrical energy use in air-conditioned offices assigned to small power. Given the publication was produced in 1996 and ICT equipment and building performance have moved on significantly over this period it has been discounted for this study.

Based on the above benchmark data work station energy consumption in the region of $22-27.45 \mathrm{kWh} / \mathrm{m}^{2} / \mathrm{yr}$. would seem to be a credible prediction for the offices under investigation.

\section{Floor Level Analysis}

The two offices spaces under consideration in this study largely utilise laptop computers with docking stations and LCD screens, occasionally a desktop lamp, destop fan, fan heater or mobile phone charger was also plugged in. Over the initial four month monitoring period average energy consumption per day at each work station and for each site was established as an indicator of the overall consumption pattern. When the average daily consumption is considered it can be seen that both sites are comparable and have similar daily profiles (see Figures $1 \& 2$ ). Across all desks the average daily consumption for the initial four month monitoring period at office A is 236 watt-hours per desk per day (for an average working day of 8.37 hours, normalised to 8 hours $=$ 225 watt-hours ) while at office B this is 307 watt-hours (for an average working day of 10.4 hours, normalised to 8 hours $=236$ watt-hours). There is a significant deviation from the mean consumption (see Figures $3 \& 4$ ) which indicates a range of behavioural 
and equipment related factors affecting consumption patterns. The standard deviation for average daily consumption at office A was 147 watt-hours per day and at office B standard deviation was 143 watt-hours per day ${ }^{2}$ indicating high but similar levels of variation. Therefore it can be said that two thirds of desks at office A consume between 89 and 383 watt-hours per day and at office B this is between 162 and 448 watt-hours per day.

In order to take account of the variation in the duration of a typical working day and equipment performance, it is necessary to consider the power density, both during working and non-working hours. The average power density during working hours at office A was 25.2 watts while at office B this was slightly lower at 22.2 watts (see Figures $3 \& 4$ ). As with the average daily energy consumption there was a significant variation in the power density at each desk over the baseline period, with a standard deviation of 14.7 and $10.3^{3}$ watts at office $\mathrm{A}$ and $\mathrm{B}$, respectively. This reflects the range of laptops, computers and screens in-use and the presence of printers, phone chargers and other small power items in various locations. The power density identified is significantly smaller than the typical benchmark of $160 \mathrm{~W}$ per desk space predicted in Energy use in Offices (DETR, 2003). Working on the basis of an average occupant density of $12 \mathrm{~m}^{2} /$ person (Gibson \& Bamidele, 2010) these power loads equate to desk level power of $2.1 \mathrm{~W} / \mathrm{m}^{2}$ and $1.85 \mathrm{~W} / \mathrm{m}^{2}$ respectively. Assuming that this equates to two thirds of overall small power (Energy efficiency in buildings, 2004) this in turn equates to a small power load of 3.15 and $2.775 \mathrm{~W} / \mathrm{m}^{2} / \mathrm{yr}$. at location A and B respectively. This compares favourably to the findings of Dunn and Knight (2005) who found small power loads of $17.5 \mathrm{~W} / \mathrm{m}^{2}$ in air conditioned UK office spaces. These figures equate to overall

\footnotetext{
${ }^{2}$ Exclude extreme cases at desks $23 \& 24$; See desk level analysis and Figure 2. (381 watts including 23 \& 24)

${ }^{3}$ Excludes extreme cases at desks $23 \& 24$; See desk level analysis and Figure 2. (17.8 including 23 \& 24)
} 
daytime workstation energy consumption of just 13.4 and $11.8 \mathrm{kWh} / \mathrm{m}^{2} / \mathrm{yr}$. based on the same occupancy density as before and an $80 \%$ attendance rate. These figures demonstrate that in comparison to industry accepted benchmarks, the locations used for the study already perform above expectation. The performance above benchmark norms of the office equipment can be attributed to the low energy procurement policy already in place at the two sites under consideration, where laptop computers have been favoured over traditional desktops. Not only is the typical power usage of laptops significantly less than that of typical desktop personal computers (20 watts versus 200 watts, on site measurement) but the power down rate of laptops tends to be in excess of that of personal computers (Kawamoto, et al. 2003) reducing the corresponding overnight wastage.

To establish where increased energy consumption or power density at a workstation can be related to equipment being left on outside of office hours it is necessary to consider the power density outside of normal working hours (when equipment not in use but may be on) at each location. This was established by taking a reading for each desk at midnight over the initial baseline period. From this it can be seen that the overnight power density for office A was 1.72 watts equating to just under $7 \%$ of the average power density, for office B the overnight power density is 5.4 watts equating to just over $24 \%{ }^{4}$ of the average (see Figures $1 \& 2$ ). Taking into account the average length of a working day in each location and despite the relatively strong performance of the sites in comparison to benchmarks, up to $23 \%$ of the overall energy consumption at desk level can be attributed to non-working hours. This indicates that there is a significant out of hour's consumption when productivity is at its lowest; it also

\footnotetext{
${ }^{4} 10 \%$ when extreme cases of desks $23 \& 24$ are excluded.
} 
supports the theory that it is possible to achieve energy and corresponding $\mathrm{CO}_{2}$ and cost reductions through behaviour change.

\section{Desk level analysis}

As the device used to monitor the energy consumption at desk level itself draws 0.8 watts it can be said that anything above this reading overnight is likely to relate to power being drawn from an additional piece of equipment. Given that a typical laptop will draw 10-60 watts (measured) and that this is likely to reduce by $22-51 \%$ when idle (Cartledge, 2008) and that a typical 17" LCD screen draws 40W (measured) reducing by a similar amount when idle it is possible by using the baseline data to estimate when workstation are powered down overnight and when they are not. Desktop lamps, heaters and mobile phone chargers were also found to be present during field surveys. Given the number of variables involved in equipment and equipment specification it was not possible to fully confirm from the data alone which specific devices if any where left drawing power out of hours.

The data, based on readings taken at midnight for each desk at office location A demonstrates that during occupied days all desks have devices left plugged in, and often turned on, with reading in excess of 0.8 watts ( 0.8 watts drawn from the monitoring device) being recorded regularly. One desk at office location B in the baseline study regularly powered down all equipment. As screens (3 watts, measured), laptops ( 2 watts, measured), mobile phone charges (0.3 watts measured) and other devices have been known to continue to draw small amounts of power when plugged in but turned off, a larger base load above that drawn by the monitoring device was set at 3 watts (equating to $26 \mathrm{kWh} / \mathrm{yr}$.). Thus, 3 watts was used to identify where power down has not occurred outside office hours. From this it was found that during the baseline monitoring period 
$72 \%$ of work stations at location A and $70 \%$ at location B powered down overnight. The identified power down rate is significantly less than that found by Kawamoto et al. (2003) which observed power down rates as high as 80-97\% in Japanese offices spaces Conversely a similar US based study found power down rates of just $36 \%$ (Roberson et al., 2006). Coupled with the initial findings of this study bringing UK power down rates in-line with those observed in the Japanese study could offer significant savings, however there is a risk that without interventions more energy could be wasted as demonstrated in the US study. Furthermore the power down rate identified reinforces the view that despite the use of efficient equipment at the sites under consideration savings due to behaviour change are still possible.

As can be seen from Figure 4, office location B has two workstations where daily energy consumption falls significantly outside the mean. Desks $23 \& 24$ feature significantly increased power densities of 91.8 and 88.7 watts respectively as compared to the site wide average of just 22.2 watts. Following the field survey desks $23 \& 24$ were found to have standard arrangements with the addition of mobile phone chargers. Comparing the power densities at these desks for working and non-working hours it was found that the workstation equipment was running twenty four hours a day, indicating the system power management was not enabled. Excluding these two desk locations from the analysis has a significant impact, reducing average daily consumption by $24 \%$ to 236.9 watt-hours (previously 307 watt-hours) thus bringing it closer to the average daily consumption at location A (236 watt-hours). This correction is carried forward into the remaining analysis. 
There is a valid argument that not all deviation from mean energy consumption can be attributed solely to behavioural issues. The specification, performance, age and configuration of the equipment being used can have a significant effect on the potential to reduce energy consumption. With this in mind, the daily consumption patterns of the top and bottom five consumers at each location were analysed (see Figures 1 \& 2). At location A it would appear that power management is enabled at all workstations under consideration as a significant overnight reduction is observed throughout. At location B three workstations do not appear to have power management enabled as minimal or no reduction is observed over night. Average power density over night compared to the average during working hours for the five lowest energy users was found to reduce by 96 and $90 \%$ at location A and B respectively. Conversely the top five energy consumes reduced by $87 \%$ and $43 \%$ at location $\mathrm{A}$ and $\mathrm{B}$ respectively. The overall average reduction at location $\mathrm{A}$ was $90 \%$ and location $\mathrm{B} 82.1 \%$. The lower reduction rate at location B can be attributed to the lack of power management in three locations. Given that older equipment with higher power densities are likely to also have less potential for energy saving in sleep or idle mode; it is difficult without further studies to fully assign the variation in observed energy consumption to equipment or behaviour. However as will be seen later in the study significant reductions can be achieved without changing work station equipment and configurations.

\section{Impact of Interventions}

Following the conclusion of the monitoring period it was found that overall reductions in line with the $20 \%$ target had been achieved. There was however significant fluctuations in the observed savings, albeit with an overall downward trend. At both locations energy use initially increased before steadily decreasing, this was somewhat unexpected, although could be interpreted as an initial reluctance to implement change. 
This is similar to the findings of the report by Cox, Higgins, Gloster and Foley (2012) on low carbon behaviours in the workplace which found a reluctance to implement change in some cases. The focus of this paper however is not to consider the psychology behind why the interventions have an impact, but to consider the potential magnitude of that impact. The behavioural aspects of why the interventions have an impact are considered in a separate paper.

Overall the average power density during working hours at occupied desks reduced by $11 \%$ at location $\mathrm{A}$ and $7 \%$ at location $\mathrm{B}$. The power down rate at location $\mathrm{A}$ increased to $79 \%$ from $72 \%$ while at location B the power down rate increased to $83 \%$ from $70 \%$, these rates compares favourably with the findings of Kawamoto et al. (2003) who observed power down rates of $80-97 \%$ in air conditioned Japanese office buildings, although further improvements may still be possible. Furthermore it would appear that $46 \%$ of users at location A and $56 \%$ of users at location B have removed any additional items overnight (mobile chargers, fans, heaters etc) and potentially unplugged computers and screen as readings of below 0.8watts (Monitoring device draws 0.8 watts) were observed on a regular basis.

The increased power down rate and corresponding energy reduction equates to an overall reduction in night time power density of 5-10\% over the follow up monitoring period at both locations. Applying the relevant working and non-working hours at both locations overall reductions in daily desk level energy consumption equate to $17.9 \%$ at location $\mathrm{A}$ and $20 \%$ at location $\mathrm{B}$. The increased overall reduction observed at location $\mathrm{B}$ over location $\mathrm{A}$ can be attributed to the $13 \%$ increase in the night-time power down rate. 
Looking more closely at the consumption of the five highest energy users at both locations it can be seen that considerable savings have been made with a 34.2 and $33.9 \%$ reduction at location $\mathrm{A}$ and $\mathrm{B}$ respectively. In contrast the five lowest energy consumers at each location have made little or no energy savings and indeed at location A an overall increase is observed (27\%), thus indicating a push to the middle. Given the locations under consideration already perform well in comparison to industry benchmarks a lack of energy savings at the lower end should not be surprising. However the push towards the middle at location A (although from a low base) is concerning and further investigation beyond the scope of this paper is required to fully understand the causes of this statistic. It could be speculated that this increase is as a result of the lower energy user's feedback demonstrating above average performance, resulting in less focus on energy savings. As demonstrated by the large standard deviation in the percentage of energy reductions achieved at each location $(50 \%+)$ there is a wide spread of results. This lack of consistency could indicate a tendency to revert back to previous habits, thus behaviour is still fluid. This would appear to support the findings of Cox et al. (2012) study on workplace initiatives for low carbon behaviour which found that persistence is needed to ensure behaviours become habit. However there is a danger of communication fatigue within this when a backlash against the preferred behaviour can be experienced. This can be hampered by high turnover rates or changes within the office structure, such changes did occur at the locations under consideration in this study and this perhaps could help to explain the level of variation observed. 


\section{Discussion}

This paper has been able to demonstrate that simple interventions can result in relatively significant energy and corresponding carbon savings. This positions the paper in line with the findings of Carrico and Riemer (2011) and Junnila (2007), where in the US and Nordic countries respectively it was demonstrated that minor interventions resulted in similar savings. The reduction in energy consumption observed ${ }^{5}$, equates to carbon savings in the order $603 \mathrm{kgCO} 2 / \mathrm{yr}$. In the context of a single building these savings are minor (relates to just 90 desks in this case), however if scaled up to the regional or national level and given the limited investment required it can be argued that the carbon savings are significant. In the context of existing buildings, where the cost of carbon savings through reductions in regulated energy can be expensive, the approach outlined can potentially offer carbon reduction of unregulated energy on a cost effective basis.

As noted by Cox et al. (2012) in the report on work place initiatives for low carbon behaviours with studies of this type there is a risk of behaviour reverting to the previous norm if mechanisms cannot be found to encourage a longer term habitual change. Ensuring the longevity of the savings achieved needs further research, however there is potential for the integration of automated mechanisms for measuring desk level consumption, encouraging savings and flagging above average usage.

To achieve such longevity, more constant monitoring and feedback is required. Such monitoring and feedback should aim to encourage energy saving habits and a

\footnotetext{
${ }^{5}$ Based on a grid carbon intensity of $443 \mathrm{~g} / \mathrm{kWh}$ (Department of Energy and Climate Change,
} 2012) 
culture of continuous improvement through behaviour change aligned with energy focused building management and procurement processes. This approach could be incorporated into the Building Management System (BMS), allowing Facilities Managers to understand energy consumption at the desk level and to measure how interventions impact upon usage patterns. Alternatively it may be possible to develop desktop applications that inform the user of their impact and energy consumption directly over a longer period. These applications could be used as a reminder of usage and to reinforce the preferred behaviour. While the monitoring and feedback mechanisms are important, procurement also has a part to play. As discussed previously, laptop computers generally use less power than traditional desktops and have been observed to result in increased overnight power down rates (Kawamoto, et al. 2003). Automatic shutdown software programmes are commercially available and as demonstrated by James (2010) can contribute to energy use reductions as part of a wider savings strategy targeting unregulated energy use.

\section{Conclusions}

This study has been able to demonstrate that there is a significant variation in desk level energy consumption within typical office spaces. It has found that even in offices with relatively efficient equipment exceeding predicted benchmark performance, there is still significant potential for further reductions through behaviour changing interventions. Up to $23 \%$ of energy usage associated with work-station equipment may occur outside of productive working hours. Additionally there is potential for significant wastage throughout the working day depending on work patterns. Power management and equipment procurement policy do offer energy savings, however even simple interventions such as an awareness of being monitored can have an impact on end user behaviour and corresponding energy use if carefully applied. 
The challenge going forward is to find mechanisms that ensure the observed energy savings are maintained over time and that there is no creep back towards previous performance. To achieve this, the behaviours that resulted in energy savings need to become habitual.

\section{References}

Abrahamse W., Steg, L., Vlek, C. \& Rothengatter, T. (2005). A review of studies aimed at household energy consumption. Journal of Environmental Psychology, 25, 273-291.

Berl, A. \& de Meer, H. (2011). An energy consumption model for virtualized office environments. Future Generation Computer Systems, 27, 1047-1055.

Bordass, W., Cohen, R. \& Field, J. (2004). Energy performance of non-domestic buildings: Closing the credibility gap. In: Proceedings from the Building Performance Congress, Frankfurt.

Carrico, A.R. \& Riemer, M. (2011). Motivating energy conservation in the workplace: An evaluation of the use of group-level feedback and peer education. Journal of Environmental Psychology, 31, 1-13.

Cartledge, C. (2008). Sheffield ICT footprint commentary: Report for SusteIT. Retrieved from http://www.susteit.org.uk

Chartered Institution of Building Services Engineers. (2008) Energy benchmarks: Technical Memoranda 46. London: CIBSE Publications. 
Chartered Institution of Building Services Engineers. (2004). Energy Efficiency in Buildings: CIBSE Guide F. London: CIBSE Publications.

Cox, A., Higgins, T., Gloster, R. \& Foley, B. (2012). The impact of workplace initiatives on low carbon behaviours: Case Study Report. Scottish Government Social Research.

Department of Energy and Climate Change. (2008). The Climate Change Act. London: The Ministry of Justice.

Department of Energy and Climate Change. (2012). Digest of United Kingdom Energy Statistics 2012. London: National Statistics Office.

Department for Environment, Food and Rural Affairs. (2012). Guidelines to DEFRA/ DECC's GHG conversion factors for company reporting: Methodology paper for emissions factors. Department for Environment, Food and Rural Affairs.

Department of the Environment, Transport and the Regions. (2003). Energy use in offices: Energy consumption guide 19. DETR Energy Efficiency Best Practice Programme.

Department of the Environment. (1996). Managing energy use. Minimising running costs of office equipment and related air-conditioning: Good practice guide 11. DOE Energy Efficiency Best Practice Programme. 
Dunn, G. \& Knight, I. (2005). Small power equipment loads in UK office environments. Energy and Buildings, 37, 87-91.

Gibson, G. \& Bamidele, J. (2010). Property in the Economy: A digest and review of key data and statistics. London: RICS.

James, P. (2010). Energy Efficient Improvement Opportunities in ICT, Evidence of the SusteIT Project. Proceedings from Energy Efficient ICT Workshop - Nottingham Trent University/

Jenkins, D.P., Singh, H. \& Eames, P.C. (2009). Interventions for large-scale carbon emission reduction in future UK offices. Energy and Buildings, 41, 1374-1380.

Jenkins, D.P., Liu, Y. \& Peacock, A.D. (2007). Climatic and internal factors affecting future UK office heating and cooling energy consumptions. Energy and Buildings, 40, 874-881.

Junnila, S. (2007). The potential effect of end-users on energy conservation in office buildings. Facilities, 25, 7, 329-339.

Kawamoto, K., Shimoda, Y. \& Mizuno, M. (2003). Energy saving potential of office equipment power managment. Energy and Buildings, 36, 915-923.

Lam, C.J., Li, D.H.W. \& Chueng, S.O. (2003). An analysis of electricity end-use in air conditioned office buildings in Hong Kong. Building and Environment, 38, 493-498. 
Menezes, A.C., Cripps, A., Bouchlaghem, D. \& Buswell, R. (2012). Predicted vs. actual energy performance of non-domestic buildings: Using post-occupancy evaluation data to reduce the performance gap. Applied Energy, 97, 355-364.

Gibson, G. \& Bamidele, J. (2010). Property in the Economy: A digest and review of key data and statistics. London: RICS.

Roberson, J.A., Webber, C.A., McWhinmey, M.C., Brown, R.E., Pinkard, M.J. \& Busch, J.F. (2006). After-hours power status of office equipment and energy use of miscellaneous plug-load equipment. Energy, 31, 2823-2838.

Cox, A., Higgins, T., Gloster, R. \& Foley, B. (2012). The impact of workplace initiatives on low carbon behaviours: Case Study Report. Scottish Government Social Research.

Vereecken, W., Deboosere, L., Simoens, P., Vermeulen, B., Colle, D., Develder, C., Pickavet, M., Dhoedt, B. \& Demeester, P. (2010). Power efficiency of thin clients. European transactions on telecommunications, 21, 479-490.

Webber, C.A., Roberson, J.A., Brown, R.E., Payne, C.T. , Nordman, B. \& Koomey, J.G. (2001). Field surveys of office equipment operating patterns. California: Lawrence Berkley National Laboratory. 
Ward, C.I. (2008). What are the energy and power consumption patterns of different types of built environment? Energy Policy, 36, 4622-4629.

Zhang, T., Siebers, P.O. \& Aickelin, U. (2011). Modelling electricity consumption in office buildings: An agent based approach. Energy and Buildings, 43, 2882-2892. 


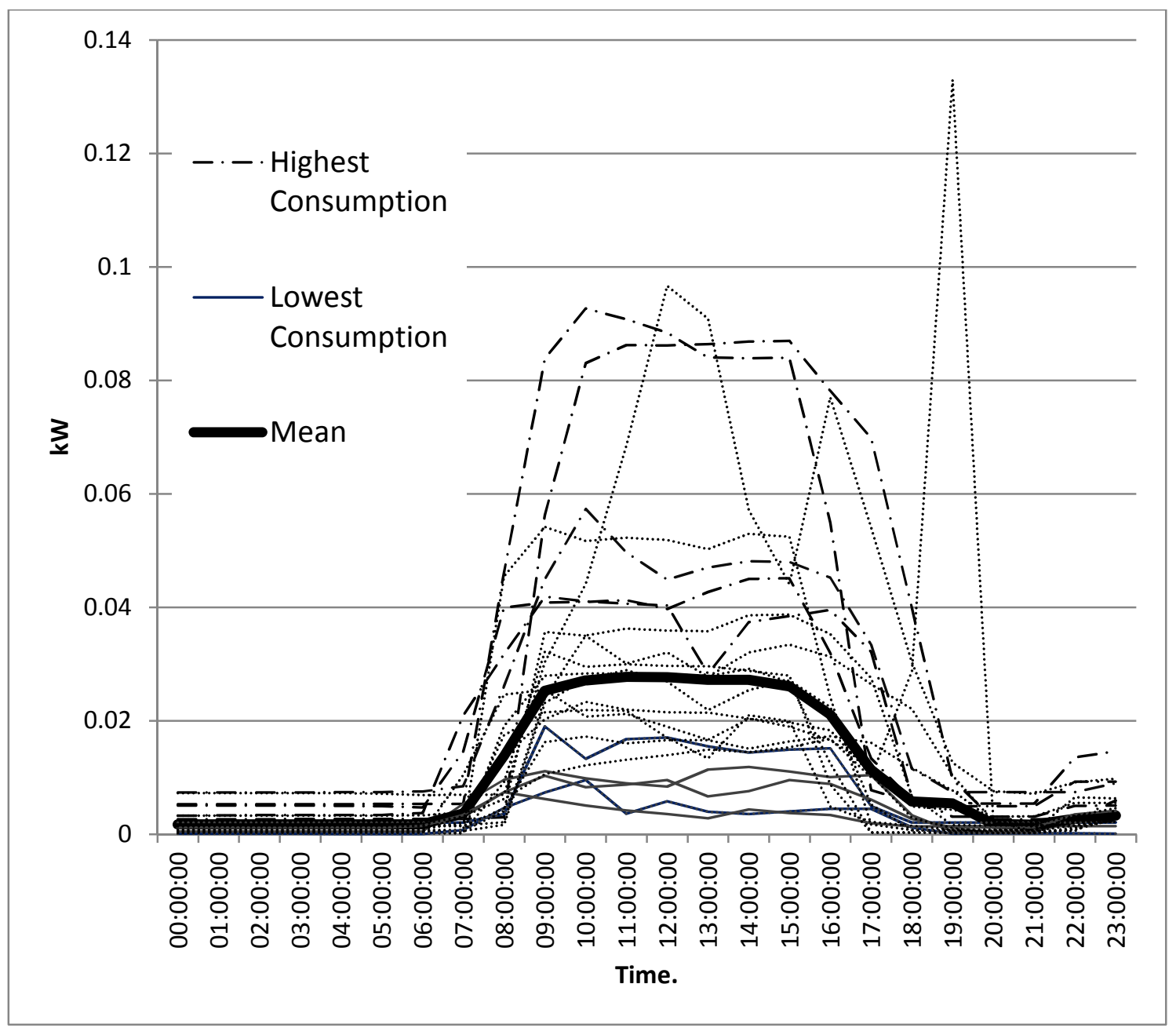

Figure 1: Daily profile location A. 


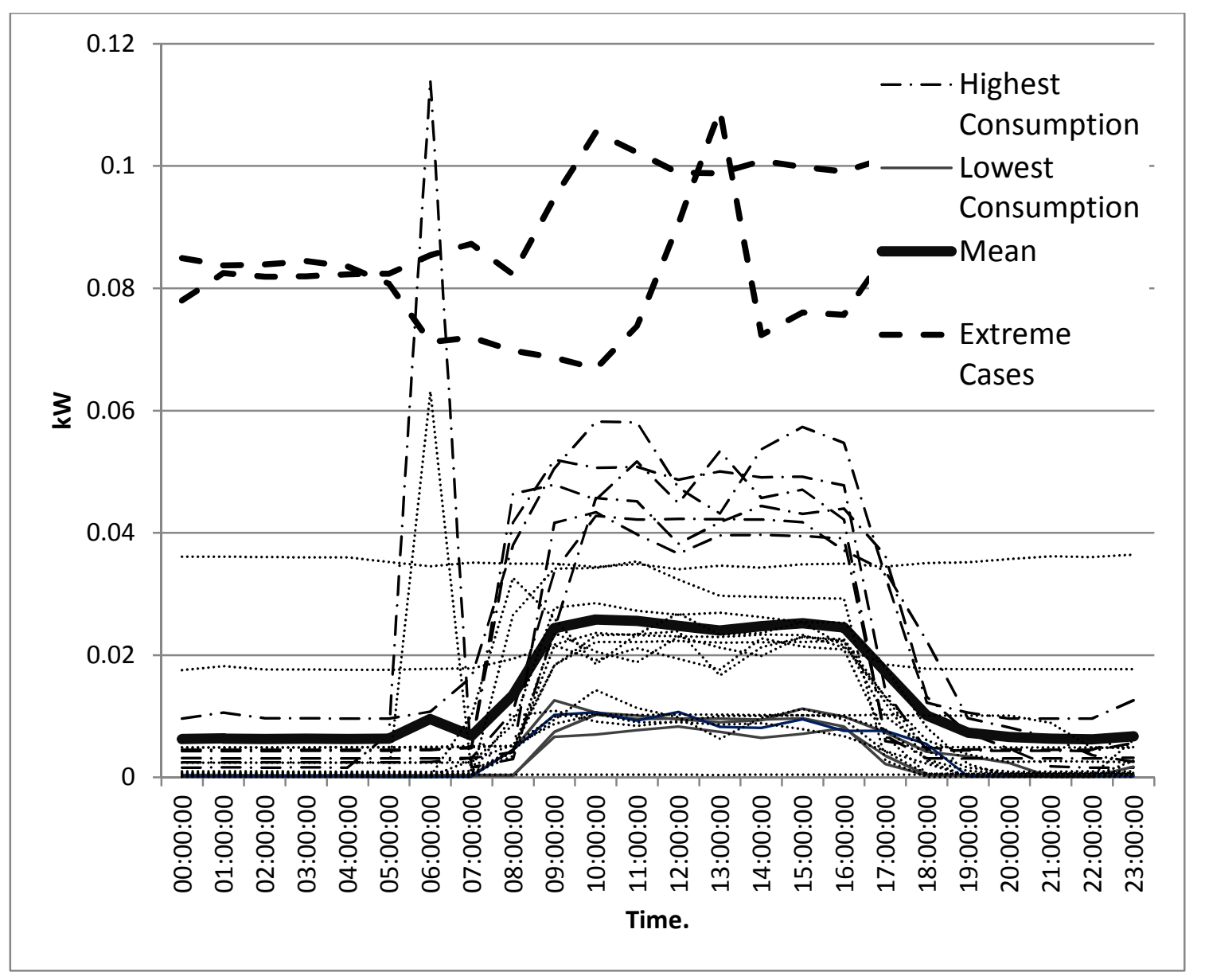

Figure 2: Daily profile location B. 


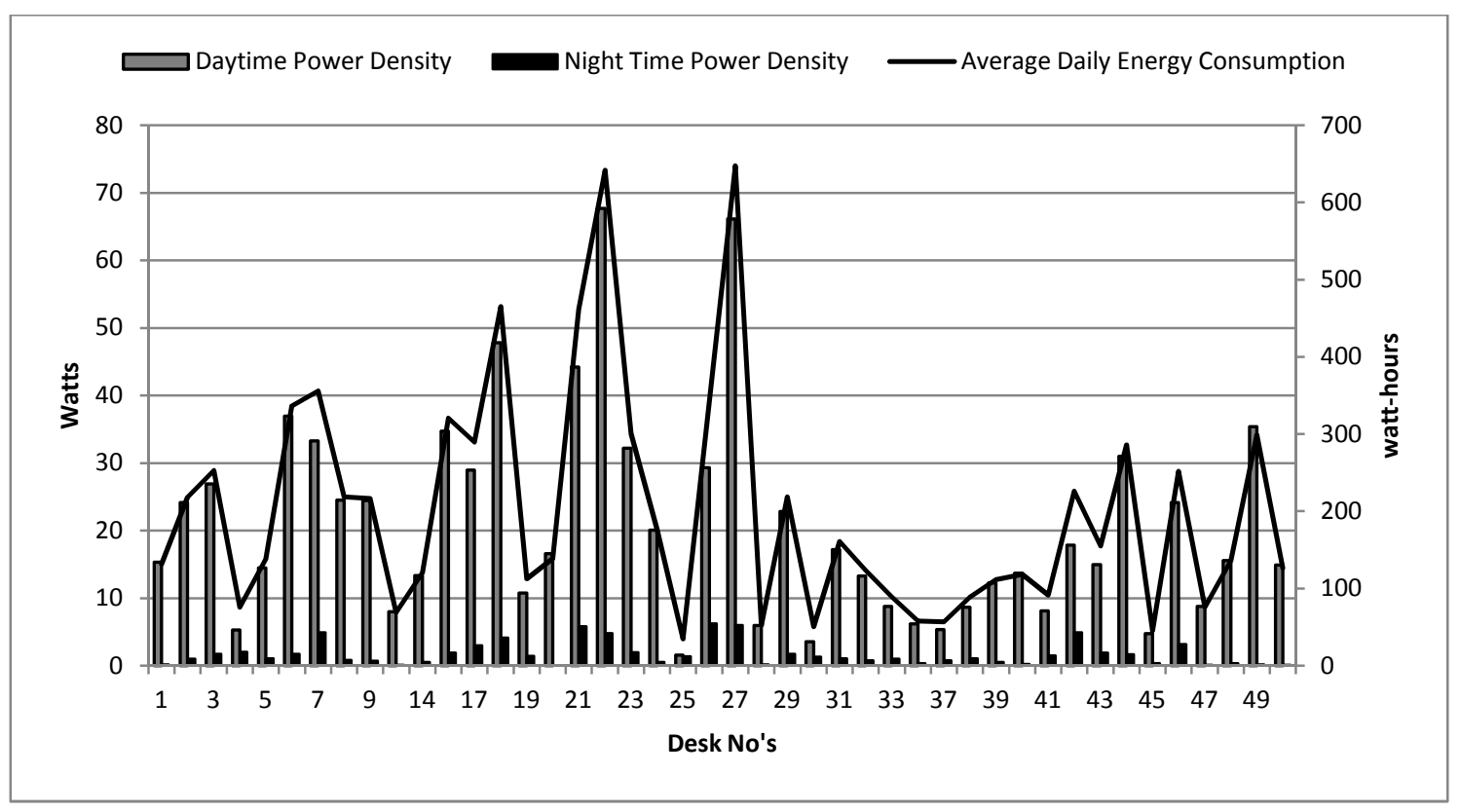

Figure 3: Power density and energy consumption location A. 


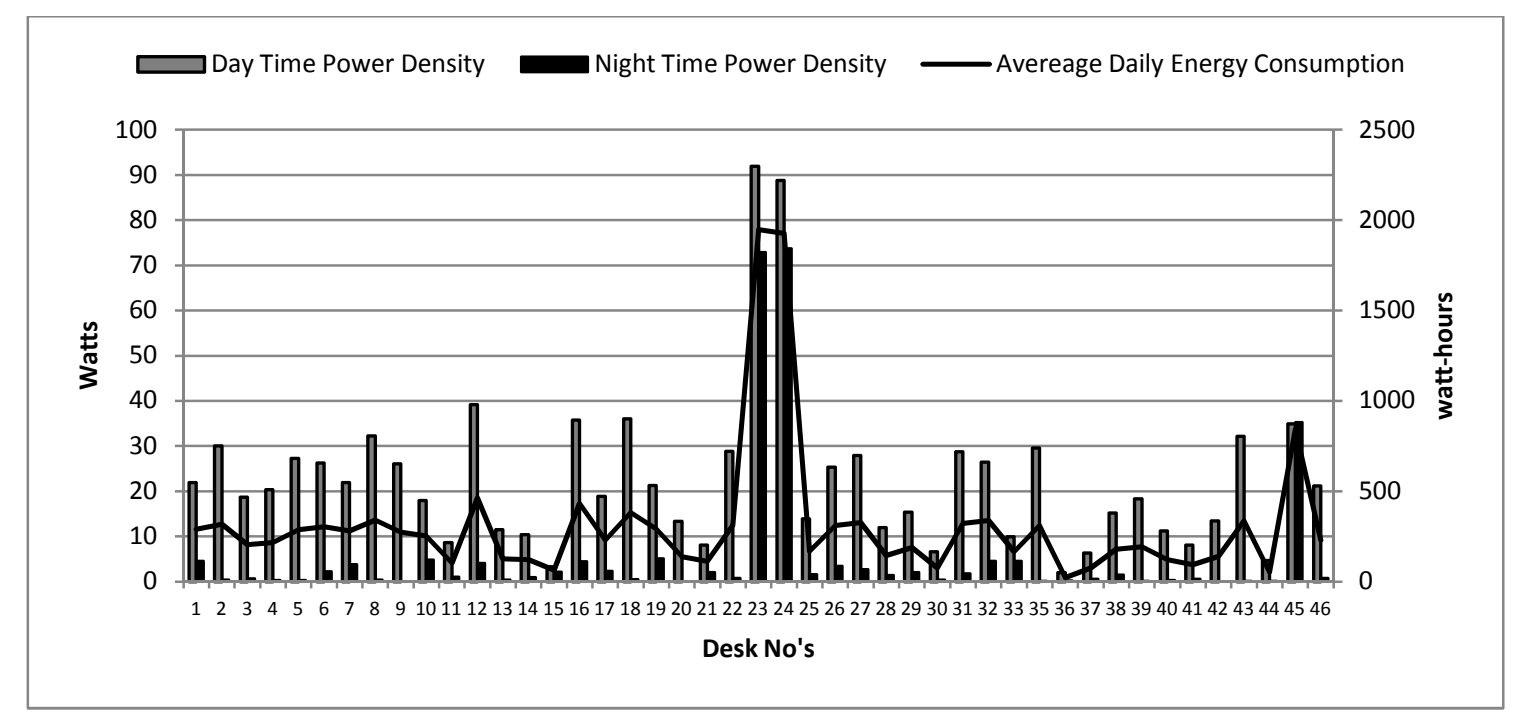

Figure 4: Power density and energy consumption location B. 